\title{
Asteroseismology of the $\beta$ Cep star HD 129929
}

\section{Seismic constraints on core overshooting, internal rotation and stellar parameters}

\author{
M.-A. Dupret ${ }^{1,3, \star}$, A. Thoul ${ }^{1, \star \star}$, R. Scuflaire ${ }^{1}$, J. Daszyńska-Daszkiewicz ${ }^{2,4, \star \star \star}$, \\ C. Aerts ${ }^{2}$, P.-O. Bourge ${ }^{1}$, C. Waelkens ${ }^{2}$, and A. Noels ${ }^{1}$
}

1 Institut d'Astrophysique et de Géophysique, Université de Liège, allée du Six Août 17, 4000 Liège, Belgium
2 Instituut voor Sterrenkunde, Katholieke Universiteit Leuven, Celestijnenlaan 200 B, 3001 Leuven, Belgium
3 Instituto de Astrofísica de Andalucía-CSIC, Apartado 3004, 18080 Granada, Spain
4 Astronomical Institute of the Wrocław University, ul. Kopernika 11, 51-622 Wrocław, Poland

Received 1 August 2003 / Accepted 25 September 2003

\begin{abstract}
We perform a detailed seismic study of the $\beta$ Cep star HD 129929. Our analysis is based on the recent derivation of six pulsation frequencies. These frequencies are unambiguously identified from the seismic modelling and the photometric amplitudes to be the radial fundamental, the $\ell=1, p_{1}$ triplet, two consecutive components of the $\ell=2, g_{1}$ quintuplet. A non-adiabatic analysis allows us to constrain the metallicity of the star to $Z \in[0.016,0.022]$. In addition, the fitting of three independent frequencies, two of which correspond to axisymmetric $(m=0)$ modes, allows us to constrain the core overshooting parameter to $\alpha_{\mathrm{ov}}=0.10 \pm 0.05$, as well as the other global parameters of the star. Finally, from the observation of the $\ell=1$ triplet and part of the $\ell=2$ quintuplet, we derive constraints on the internal rotation of this star.
\end{abstract}

Key words. stars: oscillations - stars: interiors - stars: variables: general - stars: individual: HD 129929

\section{Introduction}

Helioseismology has proven to be the most powerful tool to derive very detailed and precise information about the physics inside our Sun. Similarly, asteroseismology allows one to constrain in an unprecedented way the internal structure of stars other than the Sun by studying their oscillations. Recent efforts have been performed allowing the detection of many frequencies in different kinds of pulsating stars. Among the existing examples with solar-like oscillations, nicely summarised by Bedding \& Kjeldsen (2003), we cite the case of $\alpha$ Cen A, whose seismic studies have been recently performed by Thévenin et al. (2002) and Thoul et al. (2003a), on the base of the observations by Bouchy \& Carrier (2002). Other excellent (maybe even better) targets for asteroseismology are the $\beta$ Cep stars. Indeed, some of the $\beta$ Cep pulsation modes have a mixed pressure-gravity character allowing to strongly constrain the deep interior of these stars, and their frequency spectra are sufficiently sparse to allow easier photometric and

Send offprint requests to: M.-A. Dupret, e-mail: madupret@iaa.es

* Marie Curie Postdoctoral Fellow, European Union.

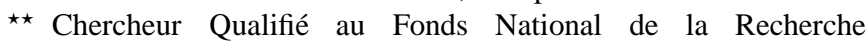
Scientifique, Belgium.

$\star \star \star$ Postdoctoral Fellow, Belgian Federal Office for Scientific, Technical and Cultural Affairs. spectroscopic mode identification compared to A-F type pulsators. The earliest seismic studies of $\beta$ Cep stars are those by Dziembowski \& Jerzykiewicz $(1996,1999)$ for respectively DD (12) and EN (16) Lac and the one by Shibahashi \& Aerts (2000) for $\beta$ Cep itself. Very recent new seismic studies of $\beta$ Cep stars were done by Thoul et al. (2003b) for EN (16) Lac and by Handler et al. (2003) for the three stars IL Vel, V433 Car and KZ Mus. The present seismic study of HD 1299229 allows us to derive even stronger constraints on its internal structure than for all the above mentioned previous cases, because more frequencies (a mixed mode $\ell=1, p_{1}$ which occurs in a triplet, and part of a quintuplet) are observed and clearly identified (Aerts et al. 2004, hereafter Paper I).

Our study is based on the observations of six pulsation frequencies of HD 129929, presented in Table 2 of Paper I. Some conclusions of the seismic analysis were already presented in Aerts et al. (2003). We give here a very complete and detailed description of our analysis. In Sect. 2, we present the models and numerical tools used in our study. In Sect. 3, we perform the photometric identification of the degree $\ell$ of these six frequencies. In Sect. 4, we present our seismic modelling of the star based on the fitting of the observed frequencies. In Sect. 5, we perform a non-adiabatic analysis of this star and derive constraints on its metallicity and overshooting. For HD 129929 it was shown in PaperI that the phase differences between the 
lightcurves in the different filters are negligible. We therefore focus on the amplitudes in this paper. Finally, in Sect. 6, we derive constraints on the internal rotation of this star, on the base of the observed triplet and parts of a quintuplet. We emphasize that HD 129929 is the first massive star for which precise constraints on the core overshooting and the internal rotation could be determined from a seismic study.

\section{Stellar models}

The stellar models in this study were computed independently with the Code Liégeois d'Évolution Stellaire (CLÉS) and with the Warsaw-New Jersey evolution code. CLÉS uses the OPAL opacities (Iglesias \& Rogers 1996) completed at low temperatures with the opacities of Alexander \& Ferguson (1994), the CEFF equation of state (Christensen-Dalsgaard \& Däppen 1992) and the atmosphere models of Kurucz (1998) as boundary conditions. The computation of the frequencies were performed by a standard adiabatic code (Boury et al. 1975). The Warsaw-New Jersey code (Paczyński 1970, Sienkiewicz, Kozłowski) computes the evolutionary main sequence models. It uses the most recent version of the OPAL opacities (Iglesias \& Rogers 1996), supplemented with Alexander \& Ferguson opacities below $\log T=3.95$, and the OPAL equation of state (Rogers et al. 1996). The effects of uniform rotation are taken into account assuming global angular momentum conservation during the evolution from the ZAMS. Linear non-adiabatic pulsations are calculated independently by two codes: the code developed by Dupret et al. (2002) and the code developed by Dziembowski (1977). In the Warsaw code, the effects of slow rotation on the oscillation frequencies are included up to the second order in the rotation velocity.

The results reported in this paper are those resulting from the Liège codes. However, we point out that the results from the Warsaw codes are very similar. A detailed comparison between the results for HD 129929, and for other stars as well, will be given in Paper III (Daszyńska-Daszkiewicz et al., in preparation).

\section{Mode identification}

HD 129929 ( $V$ magnitude 8.1, spectral type B3V) is a relatively faint variable star whose pulsation frequencies are observed only by photometry. The well-known method of photometric mode identification allows the determination of the degree $\ell$ of the different modes (see, e.g., Heynderickx et al. 1994). This method was improved by Cugier et al. (1994), who introduced the non-adiabatic eigenfunctions in it. Very recently, Dupret et al. (2003) further improved the identification method by including a more detailed treatment of the non-adiabatic pulsation in the outer atmosphere. We follow the approach by Dupret et al. (2003) in the current study. We note also that HD 129929 is a very slow rotator, so that the influence of rotation on the photometric observables (Daszyńska et al. 2002) can be neglected.

The amplitudes and phases of the six different modes as observed in Geneva filters were presented in Table 3 of Paper I.

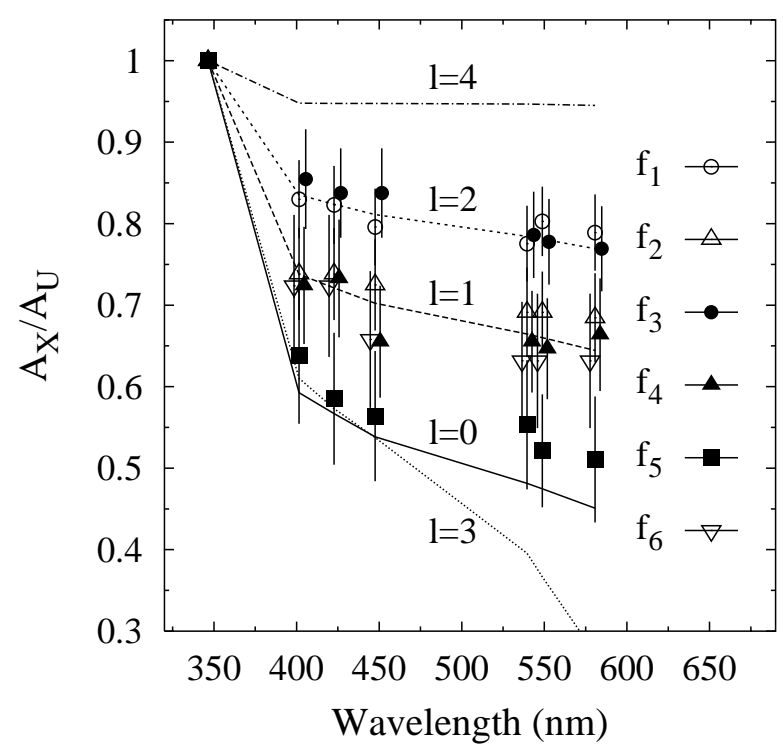

Fig. 1. Observed and theoretical amplitude ratios (Geneva photometry) for the different modes of HD 129929. The lines correspond to the non-adiabatic theoretical predictions for different degrees $\ell$ for a model with solar metallicity. The squares, circles and triangles with error bars correspond to the observed amplitude ratios for the six observed frequencies.

The observed amplitude ratios can be confronted with the theoretical ones as determined by non-adiabatic codes. We present in Fig. 1 the results obtained for a model with solar metallicity and no overshooting: $M=9.5 M_{\odot}, Z=0.02, \log T_{\mathrm{eff}}=4.3488$, $\log g=3.9094$. The lines correspond to the non-adiabatic theoretical predictions for modes of different degrees $\ell$, more precisely for the $\ell=0, p_{1}$, the $\ell=1, p_{1}$, the $\ell=2, g_{1}$, the $\ell=3$, $g_{1}$ and the $\ell=4, g_{1}$ modes. The squares, circles and triangles with error bars correspond to the observed amplitude ratios for the six observed frequencies.

We see clearly that $f_{5}$ is identified as a radial mode, the three frequencies $f_{2}, f_{4}$ and $f_{6}$ are identified as an $\ell=1$ triplet and the two frequencies $f_{1}$ and $f_{3}$ as part of an $\ell=2$ quintuplet. We performed the same analysis for a lot of other models with different metallicities and different overshooting parameters and all of them lead to the same mode identification.

\section{Frequency fitting}

The second step of our study is to determine stellar models whose theoretical frequencies fit the observed ones, in agreement with the above mode identifications. For this purpose, we have first to determine the radial order $n$ of the zonal modes. In Fig. 2, we show the evolution of the pulsation frequencies as a function of age and $\log T_{\text {eff }}$ along two evolutionary tracks. The full lines correspond to the unstable modes and the dots to the stables ones. The horizontal lines correspond to the observed frequencies of HD 129929. By confronting the theoretical models to the observations, two possible families of solutions appear. Either the radial mode $f_{5}$ can be interpreted as the fundamental mode or as the first overtone. However, interpreting it as the first overtone leads to too cold models incompatible with the observational position of the star in the HR diagram 

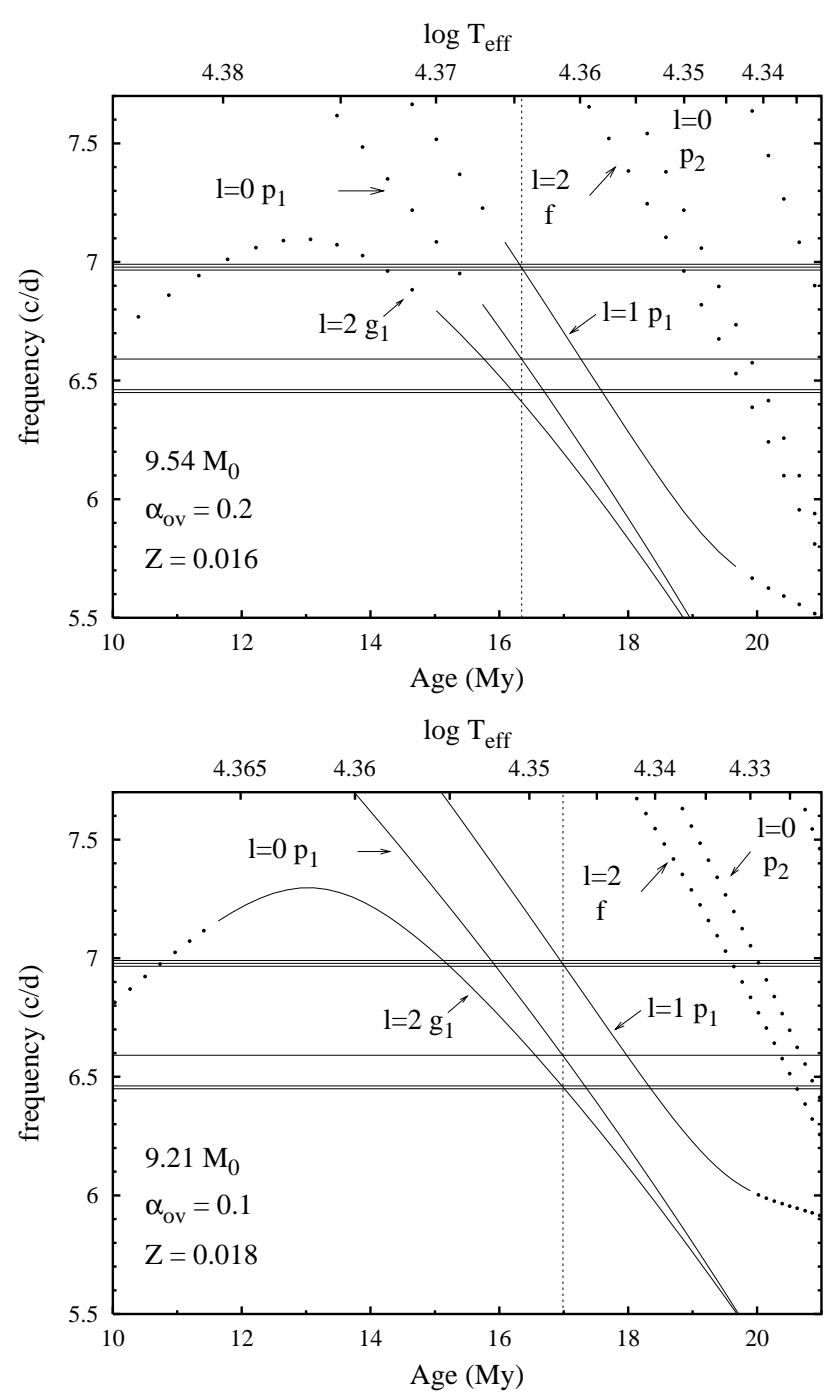

Fig. 2. Evolution of the frequencies as a function of age (Myear) and $\log T_{\text {eff }}$, for modes of degree $\ell=0,1$ and 2, for two evolutionary tracks with $M=9.54 M_{\odot}, Z=0.016$ and $\alpha_{\text {ov }}=0.2$ (top panel) and with $M=9.21 M_{\odot}, Z=0.018$ and $\alpha_{\mathrm{ov}}=0.1$ (bottom panel). The horizontal lines correspond to the observed frequencies of HD 129929. The vertical lines correspond to the models giving the best fit between the theoretical and observed frequencies of the modes $\ell=0, p_{1}$ and $\ell=1, p_{1}$. The full lines correspond to the unstable modes and the dots to the stable ones.

and with the excitation of the observed modes by the classical $\kappa$ mechanism for reasonable metallicities, as shown in Fig. 2. For this reason, we interpret it as the fundamental radial mode. Given this, the confrontation between theory and observations shows, without any doubt, that the $\ell=1$ triplet is $p_{1}$ and the two $\ell=2$ modes are $g_{1}$ (see Fig. 2).

We have two well identified axi-symmetric modes: $f_{5}\left(\ell=0, p_{1}\right)$ and $f_{2}\left(\ell=1, p_{1}\right)$ and we search for models fitting these two frequencies. We computed a number of evolutionary tracks with different values for the mass, the hydrogen and metal fractions $X$ and $Z$ and the overshooting parameter $\alpha_{\text {ov }}$. For every combination of these parameters, we can find along the evolutionary track a model whose $\ell=1, p_{1}$ mode fits $f_{2}$. Searching amongst these selected models those which

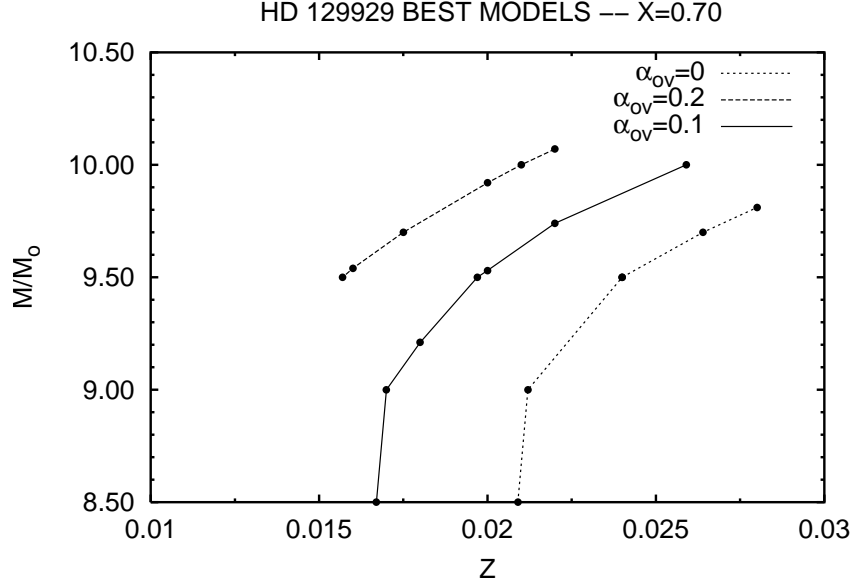

Fig. 3. Relation between the mass and the metallicity for models which fit exactly the frequencies $f_{2}$ and $f_{5}$ of HD 129929, for three families of models with different values of the core overshooting parameter: $\alpha_{\mathrm{ov}}=0,0.1$ and 0.2 . For all these models, $X=0.7$.

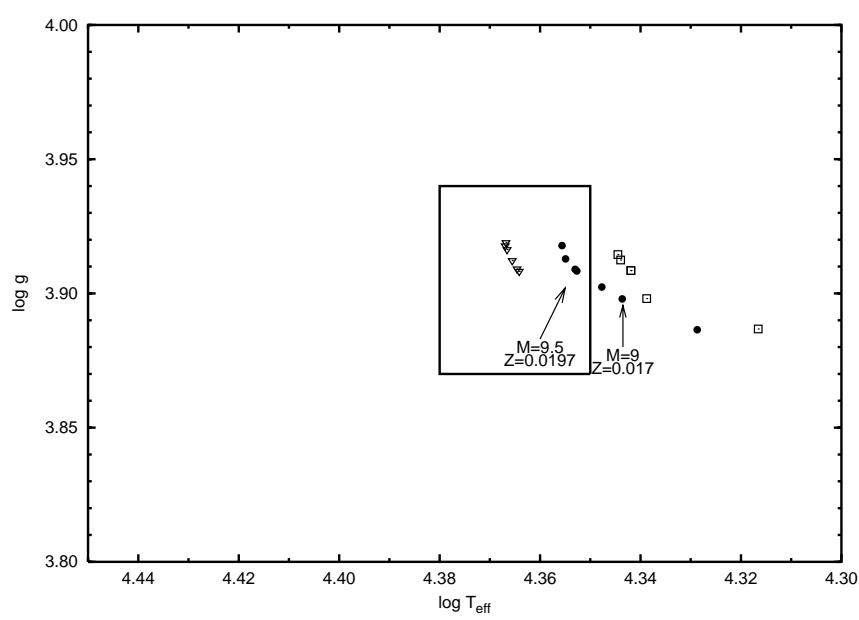

Fig. 4. Positions of the models which fit exactly the frequencies $f_{2}$ and $f_{5}$ of HD 129929 in a $\log T_{\text {eff }}-\log g$ diagram. The squares, dots and triangles are obtained for models with $\alpha_{\mathrm{ov}}=0,0.1,0.2$, respectively. The observational error box from photometry is also given.

also fit the radial mode $f_{5}$ then gives a relation between two of the four free parameters, for given values of the other two. For example, if the overshooting parameter and the hydrogen fraction are fixed, we get a relation between the mass and the metallicity for the models fitting exactly $f_{2}$ and $f_{5}$. This $M(Z)$ relation is given in Fig. 3, for three values of $\alpha_{\text {ov }}: 0,0.1$ and 0.2 and for a hydrogen fraction fixed to $X=0.7$ at the surface. The positions of these models in a $\log T_{\text {eff }}-\log g$ diagram are given in Fig. 4. In the same figure, we give also the observational error box for the effective temperature and gravity of HD 129929 derived from photometry.

We consider now the results obtained for the $\ell=2, g_{1}$ axisymmetric mode. In Fig. 5, we give the frequency obtained for this mode, as a function of the metallicity in the left panel and as a function of the mass in the right panel. The theoretical results obtained for models fitting exactly $f_{5}(\ell=0$, $\left.p_{1}\right)$ and $f_{2}\left(\ell=1, p_{1}\right)$ are represented by squares, circles and triangles for models with $\alpha_{\mathrm{ov}}=0,0.1$ and 0.2 respectively. 

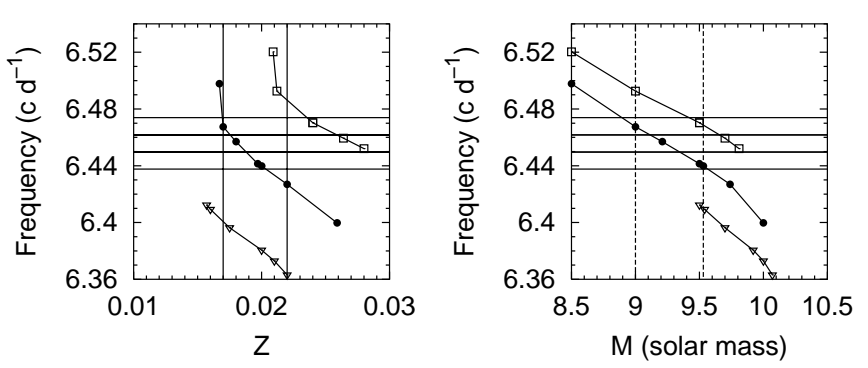

Fig. 5. Frequency of the $\ell=2, g_{1}$ axi-symmetric mode, as a function of metallicity in the left panel, and as a function of mass in the right panel. The theoretical results obtained for models fitting exactly $f_{2}$ and $f_{5}$ (Sect. 4) are represented by squares, circles and triangles for models with $\alpha_{\mathrm{ov}}=0,0.1$ and 0.2 respectively. The four possible values for the observed frequency of this mode are given by horizontal lines. The two vertical lines given in the left panel give the allowed range for the metallicity derived in Sect. 5 .

Two components $\left(f_{1}\right.$ and $\left.f_{3}\right)$ of the $\ell=2, g_{1}$ quintuplet are observed but we do not know the azimuthal order $m$ of these modes with certainty. In Paper I it was shown that $f_{1}$ corresponds very likely to the axisymmetric mode component, as two additional low-amplitude modes were found from CLEANing the periodogram (see Fig. 8 in Paper I). However, these frequency peaks were not firmly established and so we prefer not to make explicit use of them. Neglecting them, there are thus four possible values for the frequency of the $\ell=2, g_{1}$ axi-symmetric mode. These values are represented by the four horizontal lines in Fig. 5. From the left panel of Fig. 5, we see that the fitting of the $\ell=2, g_{1}$ mode imposes a relation between the metallicity and the overshooting: the higher the overshooting, the lower is the metallicity.

\section{Constraints on the metallicity and the core overshooting}

As shown in Dziembowski \& Pamyatnykh (1993), Cugier et al. (1994) and Dupret et al. (2003), the theoretical non-adiabatic predictions (growth rates, photometric amplitude ratios and phase differences) are essentially sensitive to the metallicity of the models. By requiring that the observed modes must be excited and that we get a good fit between the theoretical and observed amplitude ratios and phase differences, we obtain constraints on the metallicity. All the results presented in this section were obtained using models fitting exactly the two axi-symmetric modes, as derived in Sect. 4.

We study first the excitation of the modes. In Fig. 2, we show the evolution of the pulsation frequencies as a function of age and $\log T_{\text {eff }}$ along two evolutionary tracks. The full lines correspond to the unstable modes and the dots to the stables ones. The vertical lines correspond to the models which best fit the two observed axi-symmetric modes. The parameters of the stellar model in the top panel are $M=9.54 M_{\odot}, Z=0.016$ and $\alpha_{\mathrm{ov}}=0.2$, and in the bottom panel $M=9.21 M_{\odot}, Z=0.018$ and $\alpha_{\text {ov }}=0.1$. In both cases, we have taken $X=0.7$. As known (e.g. Dziembowski \& Pamyatnykh 1993; Pamyatnykh 1999) the range of unstable modes increases with increasing metallicity. As seen in the top panel, $Z=0.016$ is a lower bound
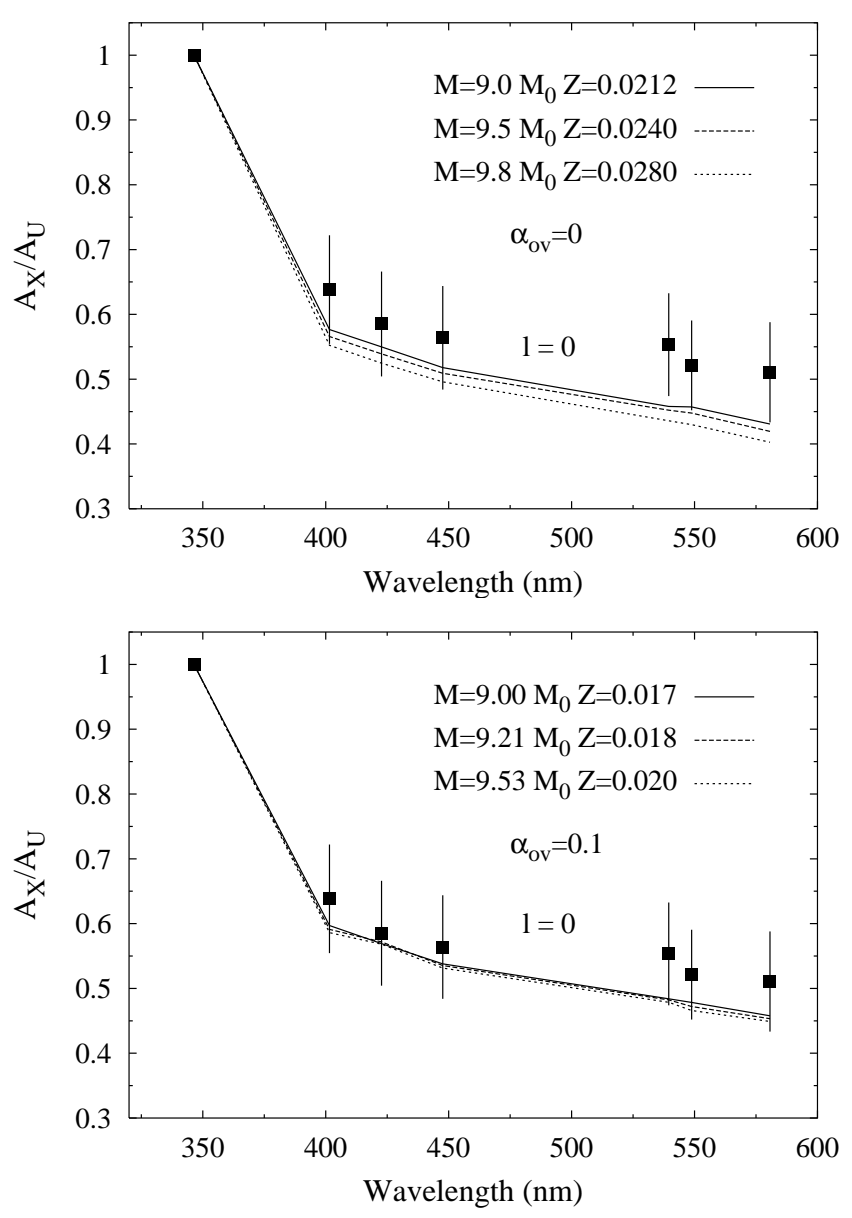

Fig. 6. Observed (squares with error bar) and theoretical amplitude ratios (Geneva photometry) for the radial mode of HD 129929. The lines correspond to the non-adiabatic theoretical predictions for different models with indicated parameters. The upper panel displays the results for the models with an overshooting parameter $\alpha_{\mathrm{ov}}=0.0$ while the models in the lower panel have $\alpha_{\mathrm{ov}}=0.1$.

below which the observed modes are no longer excited. We conclude that the models with $Z<0.016$ have to be rejected because they are not compatible with the excitation of the observed modes. On the other hand, we see in the bottom panel that, with $Z=0.018$ and $\alpha_{\mathrm{ov}}=0.1$, we obtain a model fitting exactly all the frequencies and all of them are unstable.

We also confront the theoretical and observed photometric amplitude ratios for different metallicities. It is mostly for the radial mode that the photometric amplitude ratios are sensitive to the non-adiabatic effective temperature variation (Cugier et al. 1994; Dupret et al. 2003). In order to keep a good fit between the theoretical and observed frequencies, we adopt the value $\alpha_{\mathrm{ov}}=0$ for the models with $Z>0.02$ and $\alpha_{\mathrm{ov}}=0.1$ for the models with $Z \leq 0.02$ (see Fig. 5). We show in Fig. 6 the theoretical and observed amplitude ratios in Geneva photometry for the fundamental radial mode and for two values of the overshooting parameter: $\alpha_{\mathrm{ov}}=0$ (top panel) and $\alpha_{\mathrm{ov}}=0.1$ (bottom panel). The squares with error bars are the observed amplitude ratios for the radial mode. The lines are the theoretical non-adiabatic predictions for the fundamental radial mode for different models fitting exactly the two axi-symmetric 
modes $f_{2}$ and $f_{5}$, each of them having $X=0.7$. These results are representative for the many models we have considered. We see that the discrepancy between the theoretical and observed amplitude ratios are larger for the models without overshooting than for those with $\alpha_{\mathrm{ov}}=0.1$. For the models without overshooting, those with metallicities above 0.022 are unable to explain the three observed amplitude ratios of the red filters while those with $Z<0.022$ are still consistent with the observations in the $V$ and $G$ filter. The amplitude ratios for the three $B$ filters are better explained by the models with overshooting compared to those without overshooting, although all the models lead to ratios within the error bars.

The constraints found on the metallicity of HD 129929 are thus the following. In order to have the excitation of all the observed modes, we must have $Z \geq 0.016$. Second, as shown in Paper I, the spectroscopic analysis of HD 129929 gives $Z=$ $0.018 \pm 0.004$. Third, the agreement between the theoretical and observed amplitude ratios is slightly better for the models with $Z \leq 0.02$ than for the models with $Z>0.02$.

Considering again Fig. 5, we can find constraints on the core overshooting of HD 129929. First, we see that the models with $\alpha_{\mathrm{ov}}=0.2$ must be rejected because, when fitting the frequencies, they correspond to a metallicity which is too low and incompatible with mode excitation. Second, the models with $\alpha_{\mathrm{ov}}=0.1$ are better than the models without overshooting, because their metallicities are in better agreement with the spectroscopic values derived in Paper I and they lead to a slightly better agreement between the theoretical and observed amplitude ratios. If we accept the low frequencies shown in Fig. 8 of Paper I, given that they occur at the positions where we expect the quintuplet frequencies, then we end up with $\alpha_{\mathrm{ov}}=0.1$ and $Z=0.018$, which happens to be the estimate derived from UV spectra (Paper I).

\section{Constraints on the internal rotation}

The $\ell=1, p_{1}$ triplet and two successive frequencies of the $\ell=2, g_{1}$ quintuplet are observed in HD 129929. These multiplets are interpreted as rotational splittings.

At the first order in $(\Omega / \sigma)$, where $\Omega$ is the angular rotation frequency and $\sigma$ is the angular pulsation frequency and assuming that $\Omega=\Omega(r)$, the formula for the rotational splitting has the following form:

$\sigma=\sigma_{0}+m \int_{0}^{1} \Omega(x) K_{R}(x) \mathrm{d} x$,

where $x=r / R$ and $K_{R}(x)$ depends on the considered mode (see Lynden-Bell \& Ostriker 1967; Aizenman \& Cox 1975). This formula can be used in good approximation for HD 129929, because this star is an extremely slow rotator (Paper I). In the remainder of this section we present the results obtained for one of the best stellar models of our seismic analysis. Its characteristics are given in Table 1. In what follows, we will refer to this model as the best model.

In the top panel of Fig. 7, we give the weight of the rotational splitting integral $K_{R}(x)$ (Eq. (1)), for the modes $\ell=1$, $p_{1}$ and $\ell=2, g_{1}$ and for the best model (Table 1). We see that
Table 1. Global characteristics of the "best model".

\begin{tabular}{lll}
\hline \hline$M / M_{\odot}=9.35$ & $T_{\text {eff }}=22392 \mathrm{~K}$ & $\log \left(L / L_{\odot}\right)=3.8568$ \\
$\log g=3.9054$ & age $(\mathrm{My})=16.276$ & $X_{\mathrm{c}}=0.353$ \\
$\mathrm{X}=0.7$ & $\mathrm{Z}=0.0188$ & $\alpha_{\mathrm{ov}}=0.1$ \\
\hline
\end{tabular}

Table 2. $\beta=\int_{0}^{1} K_{R}(x) \mathrm{d} x$, rotation frequency $f_{R}=\Omega /(2 \pi)$, rotation period $P_{R}$ and rotation equatorial velocity $v_{\text {eq }}$, as deduced from the multiplets $\ell=1, p_{1}$ and $\ell=2, g_{1}$, for rigid rotation models.

\begin{tabular}{lclll}
\hline \hline & $\beta$ & $f_{R}(\mathrm{c} / \mathrm{d})$ & $P_{R}(\mathrm{~d})$ & $v_{\mathrm{eq}}\left(\mathrm{km} \mathrm{s}^{-1}\right)$ \\
$\ell=1, p_{1}$ & 0.958713 & 0.012653 & 79.041 & 3.6145 \\
$\ell=2, g_{1}$ & 0.822066 & 0.014730 & 67.889 & 4.2078 \\
\hline
\end{tabular}

the $\ell=2, g_{1}$ multiplet probes the internal rotation more deeply than the $\ell=1, p_{1}$ triplet.

\subsection{Evidence of non-rigid rotation}

We begin by examining whether the two observed multiplets are compatible with a rigid rotation model. In this case, we have

$\Omega=\Delta \sigma / \int_{0}^{1} K_{R}(x) \mathrm{d} x=\Delta \sigma / \beta$.

The results obtained for the two multiplets and for the best model (Table 1) are given in Table 2 . They show clearly that the observations are not compatible with a rigid rotation model.

\subsection{Convective core rotation}

We examine now if we can derive the convective core rotation. For this, we consider the simplified case where the convective core and the radiative envelope have two different angular rotation frequencies $\Omega_{\mathrm{c}}$ and $\Omega_{\mathrm{e}}$ respectively. The rotational splitting is then given by:

$\Delta \sigma=\beta_{\mathrm{c}} \Omega_{\mathrm{c}}+\beta_{\mathrm{e}} \Omega_{\mathrm{e}}$,

where

$\beta_{\mathrm{c}}=\int_{0}^{r_{\mathrm{c}} / R} K_{R}(x) \mathrm{d} x, \quad \beta_{\mathrm{e}}=\int_{r_{\mathrm{c}} / R}^{1} K_{R}(x) \mathrm{d} x$,

and $r_{\mathrm{c}}$ is the radius of the convective core. The results obtained for the two multiplets and for the best model (Table 1) are the following:

\begin{tabular}{ccc} 
& $\beta_{\mathrm{c}}$ & $\beta_{\mathrm{e}}$ \\
\hline$\ell=1, p_{1}$ & $1.18593 \times 10^{-4}$ & 0.958595 \\
$\ell=2, g_{1}$ & $2.42282 \times 10^{-3}$ & 0.819643.
\end{tabular}

We see that $\beta_{\mathrm{c}}$ is by far too small compared to $\beta_{\mathrm{e}}$ so that no significant constraints on the convective core rotation can be derived from the observation of the two multiplets. 


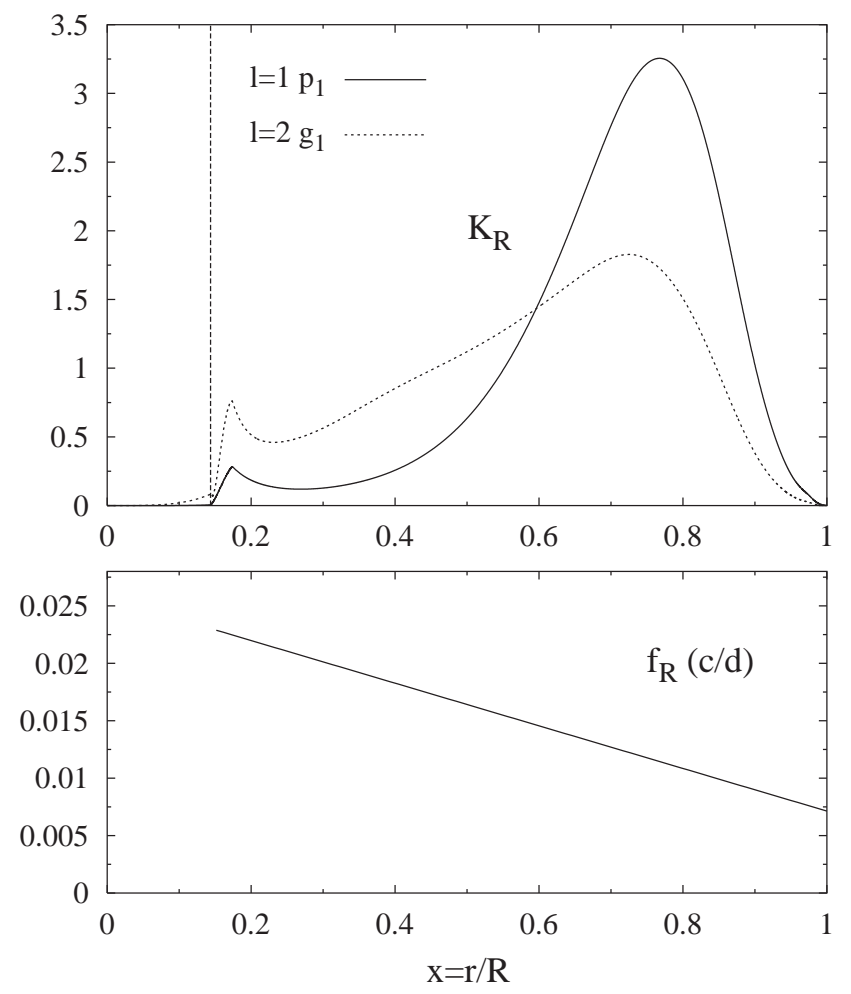

Fig. 7. In the top panel, the weight of the rotational splitting kernel $K_{R}(x)$ according to Eq. (1) is given for the modes $\ell=1, p_{1}$ and $\ell=2, g_{1}$ of the best model (Table 1 ). The vertical line is the frontier between the convective core (including the overshooting region) and the radiative envelope. In the bottom panel, the linear rotation frequency distribution $\left(f_{R}(x)=\Omega(x) /(2 \pi)\right)$, as determined in Sect. 6.3, is shown.

\subsection{Envelope rotation}

We have seen in Sect. 6.1 that the observations are not compatible with rigid rotation models. Since we have two constraints from the two multiplets, a natural approach is to consider a linear dependence of $\Omega$ with respect to $x=r / R$. Assuming

$\Omega(x)=\Omega_{0}+(x-1) \Omega_{1}$,

we get the system of two equations with two unknowns:

$\Delta \sigma_{i}=\beta_{0, i} \Omega_{0}+\beta_{1, i} \Omega_{1}, \quad i=1,2$

where the values obtained for $\beta_{0, i}$ and $\beta_{1, i}$ and for the best model (Table 1) are:

\begin{tabular}{ccc} 
& $\beta_{0}$ & $\beta_{1}$ \\
\hline$\ell=1, p_{1}$ & 0.958713 & -0.285026 \\
$\ell=2, g_{1}$ & 0.822066 & -0.336435.
\end{tabular}

The solution of Eq. (6) gives the following internal rotation frequency:

$f_{R}(x)=\frac{\Omega(x)}{2 \pi}=0.0071334-0.0185619(x-1) \mathrm{c} / \mathrm{d}$.

This corresponds to a surface rotation period of 140.21 days and an equatorial velocity of $2.037 \mathrm{~km} \mathrm{~s}^{-1}$, which is compatible with the high-resolution spectrum of the star presented in
PaperI. The linear distribution is represented in the bottom panel of Fig. 7. As expected from simple theoretical models, the rotation angular velocity is faster near the contracting core than in the outer expanding envelope.

We have assumed a linear internal rotation law in the present study. In a forthcoming paper (Daszynska-Daszkiewicz et al., in preparation, Paper III), we intend to present the internal rotation rate assuming local conservation of angular momentum.

\section{Conclusions}

In this paper we have presented a detailed non-adiabatic seismic study of the star HD 129929. We have used two independent evolution and oscillation codes and have obtained similar results with both. We have derived firm seismic constraints on the stellar parameters of the star, leading to a mass estimate with a $\sim 5 \%$ accuracy $\left(M \in[9.0,9.5] M_{\odot}\right)$ and an age determination better than some $6 \%$ as the ages of the allowed models range from 16 to 18 million years.

Several authors prefer not to account for convective core overshooting in recent evolutionary model calculations of massive main-sequence stars (see, e.g., Pamyatnykh 1999 for a discussion). The reason is that it does not seem to be a necessary ingredient to explain the statistical observed properties of such stars. However, we have found conclusive seismic evidence for the presence of overshooting with $\alpha_{\mathrm{ov}} \in[0.05,0.15]$ as our target star has an extremely low rotational velocity and so rotational effects can safely be neglected. An $\alpha_{\text {ov }}=0.17$ was proposed for a $9 M_{\odot}$ star in order for overshooting to be able to explain the broadening of the main sequence of clusters in the absence of rotational mixing (Mermilliod \& Maeder 1986). This globally derived value is consistent with our individual seismic result that overshooting occurs in HD 129929.

As is commonly done, we have adopted the standard solar mixture in our model calculations and an important open question, for any seismic analysis so far, is how robust the conclusions are if departures thereof occur. More detailed analyses of the effects of changing the mixture and/or the opacity tables will appear in a forthcoming paper (Daszynska-Daszkiewicz et al., in preparation, Paper III).

Our conclusion that core overshooting with $\alpha_{\text {ov }} \sim 0.1$ occurs in massive stars will have significant consequences for future calculations of evolutionary models of such objects and so it needs to be tested on additional stars. We stress that the $\beta$ Cep stars are by far the best laboratories to do so, because they show modes of mixed $p$ - and $g$-character and because they have no convection-pulsation interaction in their outer layers which makes them easier to interpret compared to A-F stars. However, long-term observations of $\beta$ Cep stars are necessary to cover the beat periods of their multiplets. Future asteroseismic space missions, such as COROT, Kepler and Eddington, will offer us the opportunity to improve significantly our understanding of massive star evolution, as both the precision and the sampling of the photometric data will improve by a considerable factor.

Acknowledgements. We acknowledge financial support from the Pôle d'attraction Interuniversitaire Contract P5/36, from the Prodex-ESA 
Contract 15448/01/NL/Sfe(IC), from the Fund for Scientific Research of Flanders (FWO) and from FRIA (Belgium). JDD and CA are grateful to prof. W. Dziembowski for stimulating discussions on $\beta$ Cep star seismology.

\section{References}

Aerts, C., Thoul, A., Daszyńska, J., et al. 2003, Science, 300(5627), 1926

Aerts, C., Waelkens, C., Daszynska, J., et al. 2004, A\&A, 415, 241 (Paper I)

Aizenman, M. L., \& Cox, J. P. 1975, ApJ, 202, 137

Alexander, D. R., \& Ferguson, J. W. 1994, ApJ, 437, 879

Bedding, T. R., \& Kjeldsen, H. 2003, PASA, 20, 203

Bouchy, F., \& Carrier, F. 2002, A\&A, 390, 205

Boury, A., Gabriel, M., Noels, A., et al. 1975, A\&A, 41, 279

Christensen-Dalsgaard, J., \& Däppen, W. 1992, A\&ARv, 4, 267

Cugier, H., Dziembowski, W., \& Pamyatnykh, A. 1994, A\&A, 291, 143

Daszyńska-Daszkiewicz, J., Dziembowski, W. A., Pamyatnykh, A. A., \& Goupil, M.-J. 2002, A\&A, 392, 151
Dupret, M.-A., De Ridder, J., Neuforge, C., Aerts, C., \& Scuflaire, R. 2002, A\&A, 385, 563

Dupret, M.-A., De Ridder, J., De Cat, P., et al. 2003, A\&A, 398, 677 Dziembowski, W. A. 1977, Acta Astron., 27, 203

Dziembowski, W. A., \& Jerzykiewicz, M. 1996, A\&A, 306, 436

Dziembowski, W. A., \& Jerzykiewicz, M. 1999, A\&A, 341, 480

Dziembowski, W. A., \& Pamyatnykh, A. A. 1993, MNRAS, 262, 204

Handler, G., Shobbrook, R. R., Vuthela, F. F., et al. 2003, MNRAS, 341,1005

Heynderickx, D., Waelkens, C., \& Smeyers, P. 1994, A\&AS, 105, 447

Iglesias, C. A., \& Rogers, F. J. 1996, ApJ, 464, 943

Kurucz, R. L. 1998, http://cfaku5 .harvard.edu/grids.html

Lynden-Bell, D., \& Ostriker, J. P. 1967, MNRAS, 136, 293

Mermilliod, J.-C., \& Maeder, A. 1986, A\&A, 158, 45

Paczyński, B. 1970, Acta Astron., 20, 47

Pamyatnykh, A. A. 1999, Acta. Astron., 49, 119

Rogers, F. J., Swenson, F. J., \& Iglesias, C. A. 1996, ApJ, 456, 902

Shibahashi, H., \& Aerts, C. 2000, ApJ, 531, L143

Thévenin, F., Provost, J., Morel, P., et al. 2002, A\&A, 392, L9

Thoul, A., Scuflaire, R., Noels, A., et al. 2003a, A\&A, 402, 293

Thoul, A., Aerts, C., Dupret, M.-A., Scuflaire, R., et al. 2003b, A\&A, 406, 287 